\title{
Efficient All-Optical Wavelength Converter using Saw-Tooth Pulses
}

\author{
F. Parmigiani, M. Ibsen, P. Petropoulos, D. J. Richardson \\ Optoelectronics Research Centre, University of Southampton, SO17 1BJ, United Kingdom \\ Phone: +44(0)2380593172, FAX: +44(0)2380593142,e-mail: frp@orc.soton.ac.uk
}

Wavelength conversion is a key WDM function which is used to avoid wavelength blocking, thus allowing greater flexibility in the network design. Wavelength conversion of an intensity modulated signal can be simply achieved by employing cross-phase modulation (XPM) on a CW beam placed at the desired wavelength, normally followed by a band-pass filter and a narrowband notch filter to reject the pump and the carrier frequency respectively. The notch filter in particular has to be precisely tuned to the $\mathrm{CW}$ wavelength and be extremely narrowband in order to avoid any distortions on the spectral envelope of the converted signal (see e.g. [1]). This extra complexity could be avoided using conventional band-pass filters only to select a single subcarrier band, but this usually implies non optimal $\mathrm{CW}$ extinction and thus some extra power penalties as compared to the original signal. In this paper we investigate the use of pulse shaping of the signal to be converted into a saw-tooth waveform (i.e. a pulse with leading/trailing edges of a constant intensity gradient), to improve the performance of the converted signal. Due to their linear gradient, when saw-tooth pulses are used as the XPM pump, they induce a constant frequency shift to the signal $[2,3]$. These new frequency components are highly spectrally confined and discretely separated from the $\mathrm{CW}$ carrier, thus allowing most of the wavelength converted signal energy to pass through the subsequent offset filters, thereby improving the overall optical signal to noise ratio (OSNR) and the overall system performance. Similarly, self-phase modulation (SPM) of saw-tooth pulses is more confined, giving more flexibility on the choice of the pump and signal wavelength allocations.

In our experiments we shape 1.2ps Gaussian pulses into 10ps FWHM saw-tooth pulses using a superstructured fibre Bragg grating (SSFBG) [2, 3]. Our experimental set-up is shown in Fig.1(a). The signal at the $90 \%$ port of the coupler originates from an Erbium Glass Oscillator (ERGO) at $1547.5 \mathrm{~nm}$ (the SSFBG central frequency), which generates $\sim 1.2 \mathrm{ps}$ Gaussian pulses at $10 \mathrm{GHz}$ (see Fig.1(b)). The pulses are datamodulated, shaped by the SSFBG (see Fig.1(b)) and amplified up to $20 \mathrm{dBm}$ to form the pump signal. The CW probe at $1559.1 \mathrm{~nm}$ is launched at the $10 \%$ port of a coupler. The combined signals are then launched into a highly nonlinear fibre (HNLF) (fibre parameters are reported in Fig.1(a)) to generate the XPM-induced spectral broadening. The wavelength converted signal is obtained using $0.5 \mathrm{~nm}$ optical filters detuned by $\sim 1 \mathrm{~nm}$ relative to the CW central frequency. The system has also been characterised without the SSFBG in place, though in order to avoid the build-up of excessive SPM of the pump signal, which can then overlap with the converted signal, its power was restricted to $5 \mathrm{dBm}$ when unshaped Gaussian pulses were used. Fig.1(c) shows spectral traces after the HNLF both for saw-tooth and Gaussian pulses. The figure shows that the spectral density of the wavelength shifted components is much higher when using saw-tooth pulses (see inset of Fig.1(c)), achieving an OSNR improvement of $\sim 15 \mathrm{~dB}$ relative to the unshaped Gaussian pulses. The wavelength converted signal can then be filtered using conventional band-pass filters, while maintaining good performance. Bit-error rate measurements on the wavelength converted signal (not shown here) confirm that when these filters are used with the Gaussian pulses, a power penalty of more than $6 \mathrm{~dB}$ incurs as compared to the saw-tooth case.

The authors acknowledge Furukawa Electric for the provision of the HNLF. This work was supported by EU STREP project TRIUMPH IST-027638 and FP7 Network of Excellence BONE (FP7-ICT-2007-1 216863).

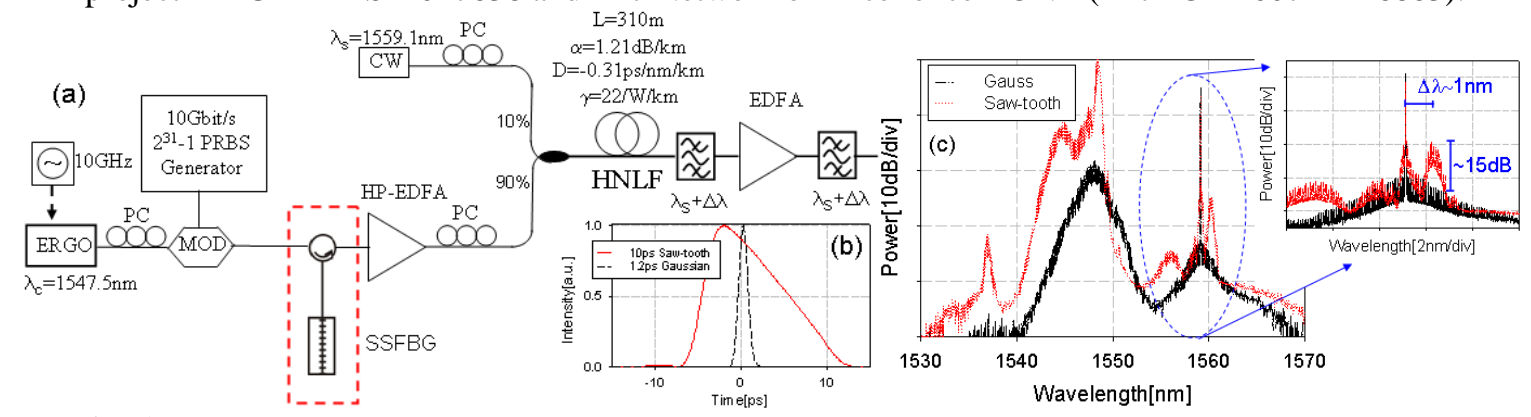

Fig. 1 a) Experimental set-up for the XPM wavelength converter. b) Intensity profile at the input/output of the SSFBG measured using a linear frequency optical gating technique. c) Spectral traces at the output of the HNLF when Gaussian or Saw-tooth pulses are used.

\section{References}

[1] B. E. Olsson, P. Ohlen, L. Rau, and D. J. Blumenthal, "A simple and robust 40-Gb/s wavelength converter using fiber cross-phase modulation and optical filtering," IEEE Photonics Technol. Lett. 12, 846-848 (2000).

[2] F.Parmigiani, P.Petropoulos, M.Ibsen, T.T.Ng, D.J.Richardson, "OTDM add-drop multiplexer using a saw-tooth pulse shaper", ECOC 2008 Brussels 21-25 Sep 2008 Th.3.C.4 (Postdeadline).

[3] F.Parmigiani, M.Ibsen, T.T.Ng, L.Provost, P.Petropoulos, D.J.Richardson "An efficient wavelength converter exploiting a grating based saw-tooth pulse shaper", IEEE Photonics Technol. Lett. 20, 1461-1463 (2008). 